Cahiers $d u$ MONDE RUSSE

\section{Cahiers du monde russe}

Russie - Empire russe - Union soviétique et États indépendants

$51 / 4 \mid 2010$

Sciences humaines et sociales en Russie à l'Âge d'argent

\title{
Alexandre Sumpf, Bolcheviks en campagne
}

\section{Emilia Koustova}

\section{(2) OpenEdition}

Journals

Édition électronique

URL : https://journals.openedition.org/monderusse/7381

DOI : 10.4000/monderusse.7381

ISSN : $1777-5388$

Éditeur

Éditions de l'EHESS

Édition imprimée

Date de publication : 25 novembre 2010

Pagination : 701-703

ISBN : 978-2-7132-2316-7

ISSN : $1252-6576$

Référence électronique

Emilia Koustova, "Alexandre Sumpf, Bolcheviks en campagne », Cahiers du monde russe [En ligne], 51/4 | 2010, mis en ligne le 09 décembre 2011, consulté le 03 septembre 2022. URL : http://

journals.openedition.org/monderusse/7381 ; DOI : https://doi.org/10.4000/monderusse.7381

Ce document a été généré automatiquement le 3 septembre 2022.

Tous droits réservés 


\title{
Alexandre Sumpf, Bolcheviks en campagne
}

\author{
Emilia Koustova
}

\section{RÉFÉRENCE}

Alexandre SUMPF, Bolcheviks en campagne. Paysans et éducation politique dans la Russie des années 1920. Paris : CNRS Éditions, 2010, 412 p.

1 Avec le livre d'Alexandre Sumpf, issu de sa thèse de doctorat, nous avons une belle occasion de suivre le slogan soviétique du milieu des années 1920 et de tourner « le visage vers la campagne» pour accompagner les bolcheviks dans leur immense entreprise, celle de la conquête de la paysannerie et de l'implantation du nouveau régime dans les villages. Il s'agit d'une occasion rare, à la fois du point de vue thématique et chronologique. À l'exception de quelques épisodes précis, tel le mouvement des correspondants ruraux (rabkory), le travail d'éducation et de propagande bolchevique dans les campagnes à l'époque de la NEP a rarement attiré l'attention des chercheurs. La violence et l'envergure des deux autres tentatives de prise du contrôle de la campagne, qui s'opèrent - de façon très différente - pendant la guerre civile, puis au moment de la collectivisation, expliquent sans doute en partie ce relatif oubli.

2 L'ambition de l'auteur - revendiquée dans l'introduction, puis mise en application dans le reste du texte - est de combiner les échelles et les focales, en allant du «centre au hameau ", de l'histoire politique et institutionnelle vers une micro-histoire, celle d'une profession et d'un milieu, d'un projet vers sa réalisation, des décideurs de Moscou aux pratiques des acteurs locaux.

3 Ce jeu d'échelles se double d'un croisement de plusieurs temporalités, la perspective historique longue étant toujours présente, avec notamment l'évocation des expériences des zemstva et des populistes russes. Sur ce dernier point, on aurait cependant souhaité davantage de précision dans l'utilisation de la référence populiste, comprise ici de façon extrêmement large, au risque d'écraser la multiplicité et la richesse des initiatives dans 
le domaine de l'instruction et de la culture populaire à la fin de l'époque tsariste. Au-delà de cette réserve mineure, une perspective historique semble en effet indispensable, tant le projet d'éducation politique - à savoir les formes qu'il prend, les appuis et les ressorts qu'il utilise, mais aussi les clichés qu'il véhicule et dont il se nourrit - est difficilement compréhensible sans la connaissance de la longue histoire, européenne et russe, du « rêve pédagogique " dont l'objectif est la création d'un homme nouveau et un transfert culturel du centre vers le terrain et notamment des villes vers les campagnes.

Continuité peut fort bien rimer avec innovation, comme le montre l'histoire de «l'éducation politique» (političeskoe prosveščenie), placée au centre de l'ouvrage. Ce concept se répand à partir de 1917, constituant une originalité forte de l'approche bolchevique qui cherche à réunir, au sein du même mouvement, instruction générale, rééducation idéologique et morale ainsi qu'activités culturelles. La complexité de l'ensemble s'exprime entre autres dans la double appartenance institutionnelle du Glavpolitprosvet, nouvelle administration qui, à partir de 1920, prend en charge le travail d'éducation politique à l'échelle du pays. Rattachée au Commissariat à l'instruction publique (Narkompros), donc au gouvernement, celle-ci dépend en même temps du parti qui, par ailleurs, possède son propre organe chargé de la propagande, Agitpropotdel.

5 L'ouvrage s'ouvre par une étude du Glavpolitprosvet pendant ses dix ans d'existence (1920-1930). Le lecteur est prévenu: il s'agit, dans un premier temps, d'une histoire institutionnelle qui contient une multitude de détails sur les remaniements de cette administration, ses rivalités avec d'autres organes de propagande, notamment ceux de l'Armée, les tentatives de définir leurs compétences ainsi que le tournant, ambigu et hésitant, dans la politique du Glavpolitprosvet, opéré au milieu des années 1920 au profit de la campagne. Cette histoire institutionnelle et politique, fortement imbriquée dans la " grande » histoire du régime soviétique et liée aux luttes pour le pouvoir, a cependant une dimension sociale et culturelle explicite, car elle pose la question des représentations et notamment de la «perception erronée de la paysannerie » qui, remontant en amont de la révolution, marque la politique bolchevique dans les campagnes.

Cette analyse institutionnelle continue dans le deuxième chapitre, en changeant cependant d'échelle pour passer sur le terrain, dans les provinces et les cantons, où un réseau local et une "verticale du pouvoir", fragile et "incomplète", du système d'éducation politique sont en train de se construire. Pour ce faire, l'auteur utilise les archives de la province de Moscou. Ce choix, même s'il procure une quantité impressionnante de sources, rend parfois problématique la généralisation et l'extrapolation des conclusions à l'ensemble du pays. Notons cependant que l'utilisation, ici comme ailleurs, d'un large éventail de sources de types différents (presse et correspondances locales, brochures...), permet de compenser en partie ces limites.

7 Avec l'arrivée sur le terrain, le deuxième grand objet de ce livre, l'izba-čital'nja, se place au centre de l'analyse. Cette invention aux fonctions et attributions parfois floues, dont le nom même pose un problème de traduction («isba-bibliothèque », "isba-salle de lecture »: aucune de ces variantes ne satisfait l'auteur qui préfère garder une transcription), a des origines assez obscures qui remontent à la période prérévolutionnaire : elle aurait fait son apparition en 1915, mais une année plus tard, on en aurait déjà dénombré plus de $9000 . .$. Quelle que soit l'extension réelle de ce phénomène, avant et pendant révolution et guerre civile, ce n'est qu'avec le renforcement progressif de l'État soviétique et l'unification opérée par le Glavpolitprosvet 
dans les années 1920, que ces modestes lieux de lecture et d'échanges, souvent tributaires des initiatives locales, commencent à être institutionnalisés, reliés dans un réseau et investis de fonctions de plus en plus précises et ambitieuses. Avec le développement des activités du Glavpolitprosvet et le tournant vers la campagne du milieu des années 1920, l'izba-čital'nja est promue " centre unifié d'éducation politique » au niveau cantonal et devient le lieu principal où s'effectue le travail d'éducation, de propagande et d'initiation à la culture dans les villages.

Dans les chapitres suivants, qui constituent la deuxième partie de l'ouvrage, on ne tourne plus « le visage vers la campagne ", on y plonge, grâce notamment à une analyse fine et originale de la place de l'izba-čital'nja dans le temps, l'espace et le quotidien villageois (ch. IV), puis grâce à une tentative d'évaluer les objectifs, activités et résultats obtenus. Il s'agit de confronter, partout, les « représentations bolcheviques » aux « réalités » du terrain, pour étudier le passage du projet à l'application, évaluer le poids du local et analyser l'impact des perceptions préexistantes. Cette réflexion passionnante révèle finalement un " échec exemplaire des ambitions trop larges des éducateurs politiques ", qui amène l'auteur à poser la question du «choc des cultures» accompagnant l'intervention bolchevique dans les campagnes.

9 La question de l'héritage culturel et du poids des représentations sociales conduit naturellement vers la dernière dimension de cette étude, qui, dans sa troisième partie, place les hommes au centre de l'analyse : responsables de l'éducation politique, diplômés des nouvelles écoles où se forge la future élite soviétique (" universités communistes »), mais surtout collaborateurs de base, les «izbači». Hommes aux parcours, formations, origines, motivations et solidarités hétérogènes, complexes et parfois contradictoires, ces responsables des izba-čital'ni permettent non seulement de pénétrer à l'intérieur du système d'éducation politique soviétique et de mieux comprendre les mécanismes de formation de la nouvelle «base» du régime, mais aussi d'approcher l'objet à la fois central et insaisissable de l'ouvrage, les paysans.

10 Malgré une volonté affichée de faire entendre leurs voix, celles-ci nous arrivent la plupart du temps étouffées, altérées ou filtrées par les sources. Dans ce jeu de cachecache, le «moujik» surgit de temps en temps des rapports ou des correspondances sous les apparences d'un izbač villageois ou encore d'une communauté locale qui cherche à utiliser les izba-čital'ni pour s'informer sur la politique bolchevique concernant la sylviculture - pour être ensuite à nouveau mis dans l'ombre par les représentations bolcheviques ou russes de la paysannerie, noyé dans la multitude de chiffres et de rapports qui projettent et façonnent une réalité, plus qu'ils ne la reflètent. Cette difficulté tient bien sûr avant tout aux sources dont les limites et les capacités déformantes sont bien connues de l'auteur; ce dernier consacre d'ailleurs plusieurs passages intéressants à discuter des spécificités et des apports de ces textes. Néanmoins, malgré ce regard critique, la «tentation statistique » reste présente. Difficile, en effet, pour un historien travaillant dans les archives soviétiques, de ne pas se laisser fasciner par l'abondance de données chiffrées qui donnent l'impression de renseigner sur la réalité dans toute sa richesse. Il s'agit là d'un problème de portée générale, et l'un des intérêts du livre est d'avoir esquissé des pistes de réponses qui pourront alimenter un débat et une réflexion ultérieure.

11 Ouvrage essentiel pour comprendre un pan mal connu de l'histoire soviétique, cette étude riche et stimulante pose ainsi nombre de questions dont l'importance dépasse ses cadres chronologiques, spatiaux et thématiques. 